\title{
Autobiographical Memory-Based Intervention for Depressive Symptoms in Young Adults: A Randomized Controlled Trial of Cognitive-Reminiscence Therapy
}

\author{
Dr David John Hallford ${ }^{1,2}$ \& Professor David Mellor ${ }^{1}$ \\ ${ }^{1}$ School of Psychology, Faculty of Health, Deakin University, 221 Burwood Hwy, Burwood, \\ Victoria 3125, Melbourne, Australia \\ ${ }^{2}$ Corresponding Author, School of Psychology, Deakin University, 221 Burwood Hwy, \\ Burwood, Victoria 3125, Melbourne, Australia, Phone: + 6139251 7777, Email: \\ david.hallford@deakin.edu.au
}

Please cite as: Hallford, D. J., \& Mellor, D. (2016). Autobiographical memory-based intervention for depressive symptoms in young adults: A randomized controlled trial of cognitive-reminiscence therapy. Psychotherapy and Psychosomatics, 85(4), 246-249. doi: https://www.karger.com/Article/Abstract/444417

Keywords: autobiographical memory; depression; cognitive-reminiscence therapy; young adults; reminiscence-based therapy; randomized controlled trial; pilot 
Increasingly, research indicates that therapies that target the recall of autobiographical memories as the core foci of interest have promise in the treatment of depression [1]. The integration of reminiscence and cognitive psychotherapies has led to the development of cognitivereminiscence therapy (CRT) [2], an effective treatment for depression in older adults [3]. In CRT, individuals engage in two kinds of reminiscence: integrative reminiscence, involving identification of continuity and meaning across experiences, and reconciliation of past negative events; and instrumental reminiscence involving the recollection of past coping and problemsolving experiences to enhance self-efficacy and perceived control, and to draw on past solutions to problems. Across a series of sessions focusing on different domains in life (e.g., turning points, stressful experiences etc.), therapists draw on both (i) cognitive models of depression and cognitive therapy techniques and (ii) stress and coping models of depression and problemsolving techniques to guide the reminiscence activities.

Although reminiscence therapies have been empirically-validated almost exclusively in older adult populations, in a recent review [4] we argued that they are likely to be equally beneficial for depressed younger adults. This is supported by studies with young adults indicating adaptive reminiscence is associated with decreases in depressive symptoms [5], and brief reminiscence activities have positive effects on self-concept, affect, and psychological resources [6].

The current study is the first to examine CRT, and reminiscence therapies generally, for treating depression in young adults. The primary outcome was depressive symptoms. Secondary outcomes were anxiety, and psychological variables proposed to be mediating variables in CRT [2]. Ethics approval was obtained from the university human research ethics committee. Twentysix young adults were recruited from a community youth mental health service for 12-25 year- 
olds and randomized, by parties external to the study, to receive CRT or usual care (see Figure one for participant flowchart). The inclusion and exclusion criteria were: 1) $18+$ years; 2) primary presenting problem of depressive symptoms, and least moderately-elevated symptoms as indicated by seven or higher on the depression subscale of the Depression, Anxiety, and Stress Scale 21-item short-form [7]; 3) not receiving treatment for depression (except for antidepressant medication stabilised for at least six weeks); 4) not at high risk of suicide or harm to others, or co-morbid psychotic or manic symptoms, or intellectual disability. At pre-intervention, threeweeks, post-intervention, and one and three-month follow-up participants completed the depression and anxiety subscales from the Depression, Anxiety and Stress Scale short-form, the Rosenberg Self-Esteem Scale, the New General Self-Efficacy Scale, the Presence subscale of the Meaning in Life Questionnaire, and a three-item version of The Life Orientation Test - Revised using a specifically-developed online portal.

Participants in the CRT group received six, weekly sessions of individual, face-to-face manualized CRT. The CRT manual was derived from a previously validated protocol [3], but adapted to combine integrative and instrumental reminiscence, be individual rather than groupbased, have age-appropriate and psychosocially-relevant content for young adults, and more specificity in therapeutic techniques based on recent research on mediators of change in reminiscence-based therapy [5]. The manual is available upon request from the first author. The therapist for the CRT group was the first author, who at the time was a registered provisional psychologist undertaking doctoral-level training in clinical psychology. An adherence checklist of the components of CRT completed after each session showed 100\% adherence, except for brief structured problem-solving, used in only $85.7 \%$ of the required sessions due to time constraints. Further details of the therapy and study protocol have been published elsewhere [8]. 
Participants in the usual care group were allocated to a registered mental health professional in the service for brief (usually 6-10 sessions) evidence-based psychotherapy. Eight participants received a cognitive-behavioural intervention (such as cognitive-therapy, acceptance and commitment therapy, or problem-solving therapy), and four received supportive counselling.

Linear mixed models (LMM) with restricted maximum-likelihood estimation were conducted to assess for effects. The data were analysed using an intention to treat approach. Mixed effects of group, time, and the interaction were modelled, and an unstructured residual covariance matrix was used to allow for correlations within participants between the repeated measures over time. Planned paired sample $t$-tests were conducted to assess changes between time-points. An alpha level of .01 was used for the $t$-tests to lower the risk of type 1 errors. A reliable change index was also used to assess change in depressive symptoms [9]

Chi-square and $t$-tests showed the groups did not significantly differ on demographic characteristics or the number of therapy sessions they received from pre-intervention to followup $(p>.05)$. The mean age was 20.8 years $(S D=1.9), 50 \%$ were female, $54 \%$ were currently studying, $61.5 \%$ were employed, and $50 \%$ had received previous support for depression. Participants had an average of $6.3(S D=2.3)$ sessions. One participant in each group entered the study on a stabilised antidepressant treatment.

Table 1 shows descriptive statistics for outcome measures and results of all LMMs. For depressive symptoms, there was an effect for group and time, but no interaction. Follow-up $t$ tests showed large effects on depressive symptoms in the CRT group at the six week and three month follow-up, but no significant differences over time in the usual care group. At six weeks, $86 \%$ of the participants in the CRT group reported reliable improvement on depressive 
symptoms, $7 \%$ showed no change, and $7 \%$ had deteriorated. In the usual care group, $50 \%$ had improved, $37.5 \%$ showed no change, and $12.5 \%$ had deteriorated. At the three month follow-up, $77 \%$ of participants in the CRT had reliably improved and $23 \%$ showed no reliable change. In the usual care group $67 \%$ reported reliable improvements, $22 \%$ stayed the same, and $11 \%$ deteriorated.

For secondary outcomes, there were main effects for time on all measures, effects for group on anxiety, self-esteem, and self-efficacy, and interaction effects on anxiety, self-esteem, and meaning in life. Follow-up $t$-tests showed large effects from baseline to the six week timepoint on all outcome measures in the CRT group, which were maintained at the three month follow-up, with the exception of anxiety symptoms. In the usual care group the only significant effect was on optimism at the six week time-point, which was not maintained at follow-up.

The results show that individually-administered CRT had large effects on depressive symptoms, which were manifest immediately following the final session, and maintained after three months. On secondary outcomes, large effects were found and maintained at follow-up. These findings provide evidence that guided reviewing of autobiographical memory with young adults has effects on a range of psychological resources: self-worth, confidence in the capacity to achieve goals and cope with challenges, the perception of meaning in one's life, and a positive future-orientation. These findings are novel in providing preliminary evidence that this intervention approach is feasible and effective for young adults. The brevity of this therapeutic intervention is advantageous. On the basis of these findings, an expanded randomized controlled trial of CRT for depression in young adults appears warranted. 
There are limitations to this study, inherent in its pilot nature. The small sample size limits the generalizability and the statistical power to detect anything but large differences. A diagnostic assessment for depression was not conducted. Future studies might compare CRT to other efficacious psychological treatments for young adult populations, such as behavioral activation or problem-solving therapy, and also account for other factors such as therapeutic alliance, allegiance effects, and perceived credibility and treatment expectancies. Mediation analyses were not conducted due to the small sample size, and future research may clarify whether changes on the secondary outcomes mediate later changes in depressive symptoms. While preliminary in nature, the finding of effects on anxiety are suggestive of transdiagnostic utility of CRT, and this might be examined in more detail. Further, examining whether changes in memory specificity might act as another mechanism of depression reduction [10] is warranted.

\section{Acknowledgements}

The authors have no conflict of interest to declare. The authors would like to thank Luke Barisic for his assistance with the online survey methodology.

Word Count: 1,274 


\section{References}

1. Dalgleish T, Werner-Seidler A.: Disruptions in autobiographical memory processing in depression and the emergence of memory therapeutics. Trends Cogn Sci 2014;18:596-604.

2. Watt LM, Cappeliez P: Reminiscence interventions for the treatment of depression in older adults. In: Haight BK, Webster JD, editors. The Art and Science of Reminiscing: Theory, Methods, and Applications. Washington: Taylor and Francis. p. 221-232.

3. Watt LM, Cappeliez P: Integrative and instrumental reminiscence therapies for depression in older adults. Intervention strategies and treatment effectiveness. Aging Ment Health 2000;4: 166-177.

4. Hallford DJ, Mellor D: Reminiscence-based therapies for depression: Should they be used only with older adults? Clin Psychol: Sci Prac 2013;20:452-468.

5. Hallford DJ, Mellor D: Autobiographical Memory and Depression: Identity-continuity and Problem-solving Functions Indirectly Predict Symptoms over Time through Psychological Well-being. Appl Cogn Psychol 2015. doi: 10.1002/acp.3169

6. Hallford DJ, Mellor D: Brief Reminiscence Activities Improve State Well-Being and SelfConcept in Young Adults: A Randomised Controlled Experiment. Memory 2015; doi:10.1080/09658211.2015.1103875

7. Antony MM, Bieling PJ, Cox BJ, Enns MW, Swinson RP: Psychometric properties of the 42-item and 21-item versions of the Depression Anxiety Stress Scales (DASS) in clinical groups and a community sample. Psychol Assess 1998;10:176-181.

8. Hallford DJ, Mellor D: Cognitive-reminiscence therapy and usual care for depression in young adults: study protocol for a randomized controlled trial. Trials 2013;14:1-6 
9. Jacobson NS, Traux P: Clinical significance: A statistical approach to defining meaningful change in psychotherapy research. J Consult Clin Psychol 1991;59:12-19.

10. Serrano JP, Latorre JM, Gatz M, Montanes J: Life review therapy using autobiographical retrieval practice for older adults with depressive symptomatology. Psychol Aging 2004;19:272-277. 


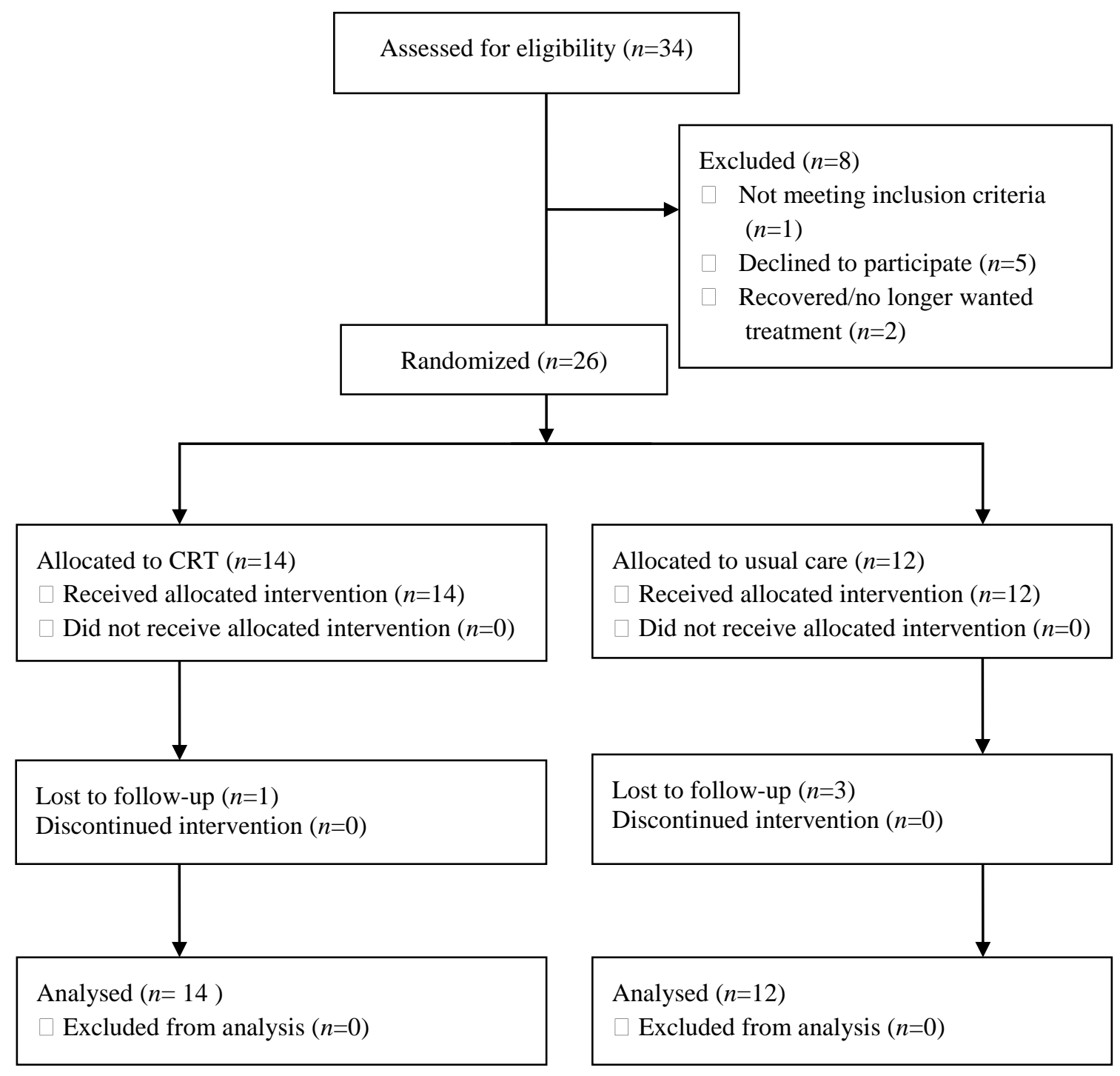

Figure 1. Participant Flowchart. 
Table 1.

Characteristics of Participants

\begin{tabular}{|c|c|c|c|}
\hline Characteristic & CRT $(n=14)$ & Usual Care $(n=12)$ & Statistics \\
\hline Age & $20.6(1.7)$ & $21.1(2.1)$ & $t=0.7, p=.494$ \\
\hline Number of Females & $8(57.1 \%)$ & $5(41.7 \%)$ & $\chi^{2}=0.6, p=.431$ \\
\hline Level of Education & & & $\chi^{2}=1.1, p=.789$ \\
\hline Primary School & $1(7.1 \%)$ & $1(8.3 \%)$ & \\
\hline High School & $9(64.3 \%)$ & $9(75 \%)$ & \\
\hline Undergraduate & $3(21.4 \%)$ & $2(16.7 \%)$ & \\
\hline \multicolumn{4}{|l|}{ Degree } \\
\hline Postgraduate & $1(7.1 \%)$ & $0(0 \%)$ & \\
\hline \multicolumn{4}{|l|}{ Degree } \\
\hline Currently Studying & $8(57.1 \%)$ & $6(50 \%)$ & $\chi^{2}=0.1, p=.716$ \\
\hline Employed & $11(78.6 \%)$ & $5(41.7 \%)$ & $\chi^{2}=3.7, p=.054$ \\
\hline Relationship Status & & & $\chi^{2}=0.1, p=.756$ \\
\hline Single & $9(64.3 \%)$ & $7(58.3 \%)$ & \\
\hline Relationship & $5(35.7 \%$ & $5(41.7 \%)$ & \\
\hline (not cohabiting) & & & \\
\hline Relationship & $0(0 \%)$ & $0(0 \%)$ & \\
\hline (cohabiting) & & & \\
\hline Married & $0(0 \%)$ & $0(0 \%)$ & \\
\hline $\begin{array}{l}\text { Previous Treatment } \\
\text { for Mental Health }\end{array}$ & $5(35.7 \%)$ & $8(66.7 \%)$ & $\chi^{2}=2.5, p=.116$ \\
\hline $\begin{array}{l}\text { Total Number of } \\
\text { Psychotherapy } \\
\text { Sessions }\end{array}$ & $6.4(1.6)$ & $6.3(3.0)$ & $t=0.2, p=.850$ \\
\hline
\end{tabular}

Note: Descriptive statistics shown as means and standard deviations in parentheses, or counts with percentages in parentheses. 
Table 2.

Means and SDs for Each Group Across Time-Points, with Paired Sample t-Tests and Effect Sizes For Six Week and Three Month Follow-up Versus Baseline Comparisons

\begin{tabular}{|c|c|c|c|c|c|c|c|}
\hline Outcome & Baseline & Three & Weeks & $\begin{array}{l}\text { One } \\
\text { Month } \\
\text { Follow- }\end{array}$ & $\begin{array}{l}\text { Three } \\
\text { Month } \\
\text { Follow- }\end{array}$ & $\begin{array}{l}\text { Baseline to Six } \\
\text { Weeks }\end{array}$ & $\begin{array}{l}\text { Baseline to } \\
\text { Three Month } \\
\text { Follow-up }\end{array}$ \\
\hline & & & & up & up & & \\
\hline \multicolumn{8}{|l|}{$\underline{\mathrm{CRT}}$} \\
\hline Depression & $12.1(3.4)$ & $6.6(4.4)$ & $5.5(4.8)$ & $4.7(3.6)$ & $5.9(4.7)$ & $p=.001, d=1.59$ & $p<001, d=1.51$ \\
\hline Anxiety & $6.2(2.9)$ & $3.3(2.8)$ & $2.1(1.5)$ & $4.0(4.4)$ & $3.8(4.4)$ & $p<001, d=1.78$ & $p=.035, d=0.64$ \\
\hline Self-Esteem & $4.3(1.4)$ & $5.8(1.5)$ & $6.8(1.1)$ & $6.6(1.3)$ & $6.4(2.0)$ & $p<001, d=1.99$ & $p<001, d=1.21$ \\
\hline Self-Efficacy & $4.9(1.6)$ & $6.0(1.2)$ & $6.7(1.4)$ & $6.7(1.4)$ & $6.6(2.0)$ & $p<001, d=1.19$ & $p=.008, d=0.93$ \\
\hline Meaning in & $3.4(1.9)$ & $5.0(2.0)$ & $6.4(2.2)$ & $5.6(2.7)$ & $6.0(2.6)$ & $p<001, d=1.46$ & $p=.001, d=1.14$ \\
\hline \multicolumn{8}{|l|}{ Life } \\
\hline Optimism & $3.7(2.0)$ & $4.7(2.1)$ & $5.5(1.9)$ & $5.7(2.2)$ & $5.3(2.5)$ & $p=.001, d=0.92$ & $p=.004, d=0.74$ \\
\hline \multicolumn{8}{|l|}{ Usual Care } \\
\hline Depression & $12.8(3.1)$ & $8.7(3.6)$ & $10.8(3.1)$ & $9.2(3.7)$ & $9.3(6.2)$ & $p=.234, d=0.64$ & $p=.218, d=0.71$ \\
\hline Anxiety & $7.1(3.6)$ & $7.0(3.6)$ & $5.9(4.6)$ & $7.0(3.4)$ & $8.6(2.9)$ & $p=.728, d=0.29$ & $p=.243, d=0.46$ \\
\hline Self-Esteem & $4.4(1.7)$ & $5.2(1.3)$ & $4.6(1.0)$ & $5.1(1.7)$ & $5.2(2.1)$ & $p=.281, d=0.14$ & $p=.188, d=0.42$ \\
\hline Self-Efficacy & $3.9(1.8)$ & $4.5(1.1)$ & $4.7(1.7)$ & $4.8(1.8)$ & $5.1(1.9)$ & $p=.529, d=0.46$ & $p=.159, d=0.64$ \\
\hline Meaning in & $4.6(2.5)$ & $4.1(2.7)$ & $4.2(2.3)$ & $4.7(2.4)$ & $4.5(2.2)$ & $p=.811, d=0.17$ & $p=.170, d=0.04$ \\
\hline \multicolumn{8}{|l|}{ Life } \\
\hline Optimism & $3.4(0.9)$ & $3.6(1.2)$ & $4.7(1.5)$ & $3.9(1.8)$ & $4.4(1.8)$ & $p=.008, d=1.05$ & $p=.021, d=0.70$ \\
\hline
\end{tabular}

Note: The possible range of scores for depression and anxiety are 0-21, and range for all other outcomes 0-10 
Table 3.

Results of Linear Mixed Models on Primary and Secondary Outcomes

\begin{tabular}{|c|c|c|c|c|}
\hline Outcome & Effect for Condition & Effect for Time & Interaction Effect & \\
\hline Depression & $F(1,24)=9.9, p=.004$ & $F(1,24)=18.6, p<.001$ & $F(1,24)=1.9, p=.146$ & I \\
\hline Anxiety & $F(1,23.4)=9.6, p=.005$ & $F(1,21.8)=4.8, p=.006$ & $F(1,21.8)=4.3, p=.011$ & $\begin{array}{l}\mathrm{CRT}<\mathrm{UC} \text { at three } \\
\text { month follow-up, } p \\
=.018\end{array}$ \\
\hline Self-Esteem & $F(1,24.1)=6.3, p=.020$ & $F(1,21.5)=12.1, p<.001$ & $F(1,21.5)=7.1, p=.001$ & $\begin{array}{l}\mathrm{CRT}>\mathrm{UC} \text { at three } \\
\text { month follow-up, } p \\
=.031\end{array}$ \\
\hline Self-Efficacy & $F(1,23.8)=10.7, p=.003$ & $F(1,20.8)=7.6, p=.001$ & $F(1,20.8)=1.7, p=.194$ & l \\
\hline Meaning in Life & $F(1,24.2)=1.0, p=.316$ & $F(1,21.8)=5.5, p=.003$ & $F(1,21.8)=4.4, p=.009$ & $\begin{array}{l}\mathrm{CRT}>\mathrm{UC} \text { at three } \\
\text { month follow-up, } p \\
=.008\end{array}$ \\
\hline Optimism & $F(1,24.3)=2.2, p=.147$ & $F(1,20.9)=6.3, p=.002$ & $F(1,20.9)=0.9, p=.475$ & l \\
\hline
\end{tabular}

Note: $\mathrm{CRT}=$ cognitive-reminiscence group, $\mathrm{UC}=$ usual care group 